\title{
Improve Performance Localization in Wireless Sensor Networks Based Received-Signal-Strength Technique and PGA
}

\author{
Virendra Jain ${ }^{1}$, Prof.Amit Thakur ${ }^{2}$ \\ M. Tech. Scholar, Department of CSE, SVCST, RGPV, Bhopal, India ${ }^{1}$ \\ Assistant Professor, Department of CSE, SVCST, RGPV, Bhopal, India ${ }^{2}$
}

\begin{abstract}
Secure localization of unknown nodes throughout a Wireless Sensing Element Network (WSN) could be an important analysis subject. Wireless detector Networks (WSNs) are networks of distributed autonomous devices which can sense or monitor Physical or environmental conditions hand and glove. WSNs face many challenges, primarily caused by communication failures, with advancements in hardware technique, communication protocols, resource efficiency, and totally different aspects. Recently, there has been a great deal of target mobile device networks, which they got even seen the event of small-profile sensing devices that are able to management their own movement. Although it has been shown that quality alleviates several issues with regard to device network coverage and property, many challenges keep. Among these, the requirement for position estimation is maybe the foremost important. Learning on localization methods for mobile wireless device networks. They provide taxonomies for mobile wireless sensors and localization, furthermore as common architectures, measure techniques, and localization algorithms. They conclude with a top level view of real-world mobile device applications that require position estimation. Describe a three stage optimization technique is planned to cut back the estimated error and finding the case of a node throughout a network. Improve performance localization in wireless detector networks based mostly received-signal-strength Technique and PGA. A resource used in WSN with wireless channels and fast channel, aware WSN and get nodes target localization is represented based parameter, wherever the channel model is incorporated into the localization scheme itself, thereby rising performance while not increasing communication overhead. Another sensible issue involving the presence of malicious sensors referred to as Byzantines is mentioned and mitigation schemes are provided. Received Signal Strength (RSST) is intricately utilized in each the 2 algorithms. The primary algorithmic rule controls the mechanical phenomenon of the mobile anchor node and uses a geometrical property to change the estimation of the situation of the unknown nodes. old method RSST more error but our proposed method less error.
\end{abstract}

Keywords: Wireless sensor Network, security, localization, mobile sensors, anchor nodes, Localization error, received signal strength technique, PGA

\section{INTRODUCTION}

Wireless Detector Network (WSN) includes a sizable amount of detector nodes that are deployed in some areas. they will be utilized in several applications, i.e. battlefields, hearth vulnerable, security areas, observation surroundings conditions, following functions, event detection et al.. Among most of those applications, location data of detector nodes is crucial. Thus, it's necessary to get the placement data, that is termed as localization. Location identification or localization in Wireless device Network refers to estimating the position or abstraction co-ordinates of Wireless device Nodes. Localization may well be done either manually or by victimization GPS. normally the localization method equip every node with a world positioning system, that isn't effective thanks to size, value and power consumption constraints. The limitation of GPS is that it can't be enforced in dense forests, mountains that block the road of sight from satellites. Therefore, several localization algorithms introduced to resolve the localization drawback. Localization rule in WSN use special nodes referred to as landmarks or anchors to estimate the placement of alternative nodes employing a priori data of the special node co-ordinates. Some special nodes referred to as anchors being awake to their position and alternative nodes referred to as unknown nodes measures the gap from special nodes to estimate their positions. [1]

Localization is one in every of the foremost vital topics in Wireless detector Networks (WSNs) since several basic techniques in WSNs, e.g., location-based authentication need the positions of unknown nodes. Also, the positions of unknown nodes play a crucial role in several WSNs applications, like observation applications embody environmental observation, health observation, and following applications embody pursuit objects, animals, humans, and vehicles [2]. Specifically for a few applications, e.g., military applications like field of battle police work or environmental applications like fire detection, incorrect positions might cause severe consequences, e.g., wrong military selections on 
the field of battle and false alarms to folks thus, the problems of secure localization should be self-addressed in WSN[3]. detector network deployments are usually determined by the applying. Nodes may be placed during a grid, randomly, encompassing AN object of interest, or in incalculable alternative arrangements. In several things, AN best preparation is unknown till the detector nodes begin assembling and process information. For deployments in remote or wide areas, rearranging node positions is usually impossible. The design of economical protocols for detector networks has been a really active analysis space in recent years. A elementary drawback in designing device networks is localization - determinative the locations of the sensors. This info is helpful in several contexts - it should be used for cluster, routing and for mapping the sector being perceived. In straightforward terms, localization is mechanism for locating spatial relationship between objects. decisive the physical location of sensors when they need been deployed is thought because the drawback of localization. Sensors responsive to their position also can improve routing potency by selective flooding or selective forwarding knowledge only within the direction of the destination. detector nodes might not have AN individual identifiers or addresses. the placement of the detector could also be a part of different address of the sensors. numerous algorithms that use the placement as a part of the address are propose[4]

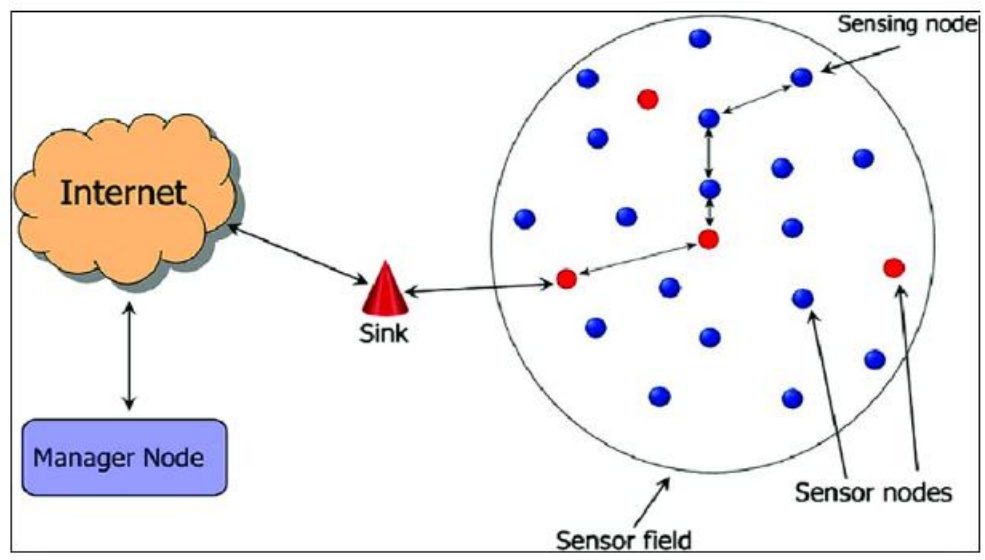

Fig1 WSN communication design

Localization is a very important analysis drawback in several systems like measuring instrument, sonar, the world positioning system, wireless networks, psychological feature radio networks transmission systems, and wireless device networks (WSNs). supply localization employing a network of sensors or wireless nodes has sweeping applications, e.g., wireless location services; search, rescue, and navigation in harsh environments; autonomous vehicles; net of Things; and watching and police work for security and defense. As a result, this analysis topic has received continuous interest and has been investigated widely [5]

\section{LITERATURE SURVEY}

In Niu et al. [6] this paper, an overview of recent developments in received-signal-strength (RSS)-based localization in wireless sensor networks is presented. Several important practical issues and their solutions are discussed. To save communication bandwidth and sensor energy, a maximum-likelihood estimator based on quantized data is presented along with its corresponding CrameÂr $\square$ Rao lower bound (CRLB) and optimal quantizer design schemes. For further system resource savings, an iterative sensor selection approach is presented to activate only the most informative sensors, by maximizing the mutual information or minimizing the posterior CRLB at each iteration. For a resource constrained WSN with imperfect wireless channels, channel-aware target localization is described, where the channel model is incorporated into the localization scheme itself, thereby improving performance without increasing communication overhead. Another practical issue involving the presence of malicious sensors called Byzantines is discussed and mitigation schemes are provided. A recent coding-theory based approach which is both computationally inexpensive and robust to such malicious attacks is also discussed.

Harikrishnan.G et al.[7] Sensor localization has become an essential requirement for realistic applications over Wireless Sensor Networks (WSNs ). As such localization scheme designed for mobile sensor networks is necessary. Here the objective is to design a localization scheme to improve the localization accuracy by using localization scheme Improve d Monte -Carlo Localization (IMCL). In this method, all se ns or nodes are mobile, normal node collects the locations of its one -hop and two-hop anchor nodes via message exchange, and constructs a ne w possible location se $t$ in e ach time s lot where the normal node may locate. The normal nodes without location information can estimate their own locations by gathering the positions of location -aware nodes (anchor nodes ) and the one - hop normal nodes whose locations are estimate $\mathrm{d}$ from $\mathrm{t}$ he anchor nodes. In e ach time $\mathrm{s}$ lot e ach normal node executes these three phases once and gets its estimate d location. Our simulation result shows that the localization error using this scheme is 
lower than $\mathrm{t}$ he previous schemes such as MCL, MSL and bounded box schemes under various mobility models and moving speeds.

Balasubramanian S et al.[8] Localization is an essential and important research issue in Wireless Sensor Networks (WSN).Most localization schemes focus on static sensor networks. However, Mobile sensors required in some applications to acquire all the relevant dat. As such, a localization scheme defined for mobile sensor networks is necessary to track the moving nodes In this paper, we propose a localization scheme, the normal nodes without location information can estimate their own location information can estimate their own locations by gathering the positions of location aware nodes (anchor nodes) and the one-hop normal nodes whose locations are estimated from the anchor nodes. In addition, we propose a scheme that predicts the moving direction of sensor nodes to increase localization accuracy. Simulation results show that the localization error in our proposed scheme is lower than the previous schemes in various mobility models and moving speeds.

Du et al. [9] In wireless sensor networks (WSNs), sensors' locations play a critical role in many applications. Having a GPS receiver on every sensor node is costly. In the past, a number of location discovery (localization) schemes have been proposed. Most of these schemes share a common feature: they use some special nodes, called beacon nodes, which are assumed to know their own locations (e.g., through GPS receivers or manual configuration). Other sensors discover their locations based on the reference information provided by these beacon nodes. Most of the beacon-based localization schemes assume a benign environment, where all beacon nodes are supposed to provide correct reference information. However, when the sensor networks are deployed in a hostile environment, where beacon nodes can be compromised, such an assumption does not hold anymore. In this paper, we propose a general scheme to detect localization anomalies that are caused by adversaries. Our scheme is independent from the localization schemes. We formulate the problem as an anomaly intrusion detection problem, and we propose a number of ways to detect localization anomalies. We have conducted simulations to evaluate the performance of our scheme, including the false positive rates, the detection rates, and the resilience to node compromises.

In Sheu J.P et al.[10] the literature, there exist many solutions to locating moving objects based on different localization issues for wireless sensor networks (WSN). Sensor networks are expected to revolutionize information gathering, processing and dissemination in many diverse environments. Existing localization algorithms can be categorized as either range-based or range-free schemes. Range - based schemes are not suitable for WSN because of their irregularity of radio propagation and their cost of additional devices. In contrast, range-free schemes do not need to use received signal strength to estimate distances. They only need simple and cheap hardware and are more suitable for WSN. However, existing range-free schemes are too costly and not accurate enough or are not scalable. To improve previous works, a fully distributed range-free localization scheme for WSN [12] is presented, based on the assumption that only a few sensor nodes, called anchors, know their locations, and the remaining (normal) nodes need to estimate their own locations by gathering nearby neighboring information. The improved grid-scan algorithm is used here to find the estimated locations of the normal nodes and a vector-based refinement scheme to improve the accuracy of the estimated locations.

Md. Tareq Adnan et al.[11] Localization is defined as the problem of determining the geographical locations of nodes in a wireless ad hoc or sensor network. Networks typically have some nodes (called seeds) that know their locations (using GPS or other means). Other (non-seed) nodes compute their locations by exchanging messages with nodes within their radio range. Several algorithms have been proposed for localization in different scenarios. Algorithms have been designed for networks in which each node has ranging capabilities (i.e., can estimate distances to its neighbors). Other algorithms have been proposed for networks in which no node has such capabilities. Some algorithms only work when nodes are static. Some other algorithms are designed specifically for networks in which all nodes are mobile. In this paper we propose a range-based localization algorithm RMCB for wireless sensor networks. Our algorithm uses a small fraction of seeds. It also makes use of the received signal strength measurements that are available from the sensor hardware. Our algorithm works well when nodes are static or mobile. We demonstrate using empirical data from sensor hardware (Texas Instruments EZ 430-RF2500) and simulations that RMCB outperforms a very good range-free algorithm WMCL in terms of localization error and has a similar computational complexity to WMCL.

Xinwei Wanga et al. [12] A multilateration algorithm is presented in this work in order to realize a precise localization of the sensor nodes in wireless sensor networks in logistic applications. RSSI (Received Signal Strength Indicator) is used for distance measurements between sensor nodes. The developed algorithm is simulated and afterwards implemented in a real sensor network for food transportation logistic. The test results show that the multilateration algorithm with RSSI reaches the requirements of logistic applications.

B. Sriniva al. [13], was proposed that in WSN the localization was an essential issue because many applications require sensor nodes to know their locations with a high degree of precision. An optimal path planning method for the mobile anchors based on localization. The proposed path planning method determined the location of the individual sensor nodes with the help of mobile anchor nodes. It ensured that the trajectory of the mobile anchor nodes minimized the localization error and guaranteed that all of the sensor nodes could determine their locations. Then, the PSO algorithm 
determined the trajectory of the mobile anchor nodes. The path planning strategy method performed in both smaller localization error and a high percentage of localized sensor nodes by PSO.

S.H. Hong et al.[14]. This paper describes a localization algorithm necessary for building small-sized network's position reporting system using wireless sensor network. In existing sensor networks, much of the localization algorithm is devoted to making position estimations by learning a minimum of 3 position values of anchor node that's aware of absolute position value under the environment where sensor nodes are fixed. The proposed algorithm has an indefinite traveling direction and has shown in performance analysis that it is possible to estimate positions even with a small number of anchors. Also, by using the implemented board, although practicality has been proven with implementation of realistic distance measurement through distance measurement using RSSI(Received Signal Strength Indication) and traveling distance measurement using acceleration sensor.

R. Shubair et al.[15]. the distance-based localization techniques are surveyed for WSNs. It is impossible to present a complete review of every published algorithm. Therefore, ten representative distance-based localization algorithms that have diverse characteristics and methods are chosen and presented in detail. The authors outline a tiered classification mechanism in which the localization techniques are classified as distributed, distributed-centralized, or centralized. Generally, centralized localization algorithms produce better location estimates than distributed and distributedcentralized algorithms. However, much more energy is consumed in the centralized algorithms due to high communication overheads for packet transmission to the base station. Distributed-centralized localization algorithms are always used in cluster-based WSNs, which can produce more accurate location estimates than distributed algorithms without significantly increasing energy consumption or sacrificing scalability. Localization of sensor nodes is an important aspect in Wireless Sensor Networks (WSNs). This paper presents an overview of the major localization techniques for WSNs. These techniques are classified into centralized and distributed depending on where the computational effort is carried out. The paper concentrates on the factors that need to be considered when selecting a localization technique. The advantages and limitation of various techniques are also discussed. Finally, future research directions and challenges are highlighted.

\section{PROBLEM FORMULATION}

Localization is one among the most drawback in WSN. to resolve this downside several localization techniques are used. In WSN localization technique may be explained in 2 section that are go section \& Position section. In go section, node live their distances from the anchors victimisation several algorithms as Received signal strength, AOA (angle of arrival), TOA (time of arrival) etc. In estimation section the node estimate their position mistreatment the go data. this is often done either by mistreatment geometric approach which provides the precise resolution or by victimisation the improvement approach that reduced the error in locating the coordinates of the device node (unknown node). several researchers had approached the localization drawback from totally different perspective. Here we tend to target the localization technique supported distance measurements and reduced the localization error between the calculable location of the device node.

\section{MATLAB TOOL}

The Performance analysis of MATLAB version (R2013a) i.e. used for this thesis Implementation of data mining provides processor optimized libraries for fast execution and computation and performed on input cancer dataset. It uses its JIT (just in time) compilation technology to provide execution speeds that rival ancient programming languages. it should additional advantage of multi core and computing device computers, MATLAB provide many multi rib pure mathematics and numerical perform. These functions automatically execute on multiple procedure thread during a) very single MATLAB, to execute faster on multicourse computers. throughout this thesis, all inflated economical data retrieve results were performed in MATLAB.MATLAB is that the high level language and interactive surroundings used by numerous engineers and scientists worldwide. It lets the explore and visualize ideas and collaborate across entirely completely different disciplines with signal and image method, communication and computation of results. MATLAB provides tools to accumulate, analyze, and visualize info, modify you to induce insight into your info during a very division of the time it would take exploitation spreadsheets or ancient programming languages. it should document and share the results through plots and reports or as unconcealed MATLAB code. MATLAB (matrix laboratory) may well be a multi paradigm numerical computing situation and fourth generation linguistic communication. it's developed by branch of knowledge work; MATLAB permits matrix strategy, plotting of perform and data, implementation of algorithmic program, construction of user interfaces with programs. MATLAB is supposed primarily for mathematical computing; associate no mandatory tool box uses the MuPAD symbolic engine, allowing access to symbolic computing capabilities. it's simulating on mat laboratory seven. And for this work we have a tendency to use Intel one. $4 \mathrm{GHz}$ Machine and package window7, window-xp etc. MATLAB version thirteen may well be a high-level technical calculate language and interactive surroundings for rule development, data visual image, records analysis, and numeric computation Mat laboratory may well be a code 
Vol. 8, Issue 6, June 2019

program that allows you to undertake and do info manipulation and visual image, calculations, branch of knowledge and programming.

\section{RESULT ANALYSIS}

In the field of research wireless sensor networks and determines several challenges. Find accuracy and minimize error in wireless sensor networks communication and optimum answer.

Table 1 Design Simulation Network

\begin{tabular}{|l|l|}
\hline Parameter Name & Set Parameter Values \\
\hline Length(m) $\mathrm{x}$ Height(m) $\mathrm{x}$ Width(m) & $105 \times 105 \times 105$ \\
\hline Approximate Distance Calc. (\%) & 5 \\
\hline Approximate Angle Calc. (\%) & 5 \\
\hline Population Size & 75 \\
\hline Maximum Iterations & 105 \\
\hline Number of Nodes & $15,16,18$ \\
\hline
\end{tabular}

(a) Error Estimation Graph Analysis

In expertmention1 supported 15 nodes error estimation values compare RSST and PGA show lead to graph and PGA realize error values 17.7756 and RSST realize 20.3453 .overall notice PGA Technique best resolution in wireless sensing element network

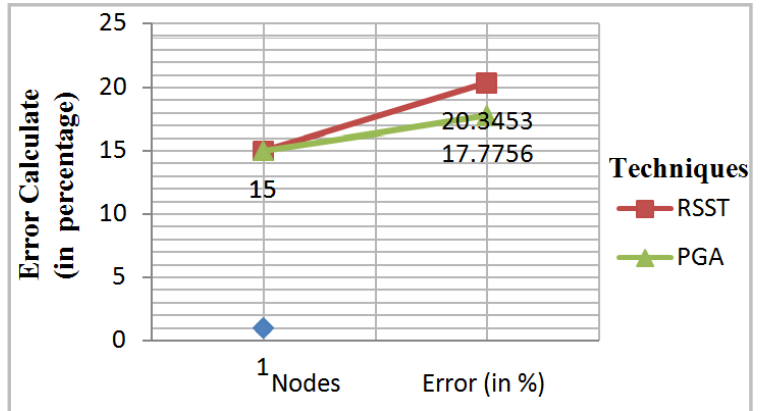

Fig 2 Error Performance comparison between RSST and PGA in expertmention1: 15 nodes

\section{(b) Total Execution Time Graph Analysis}

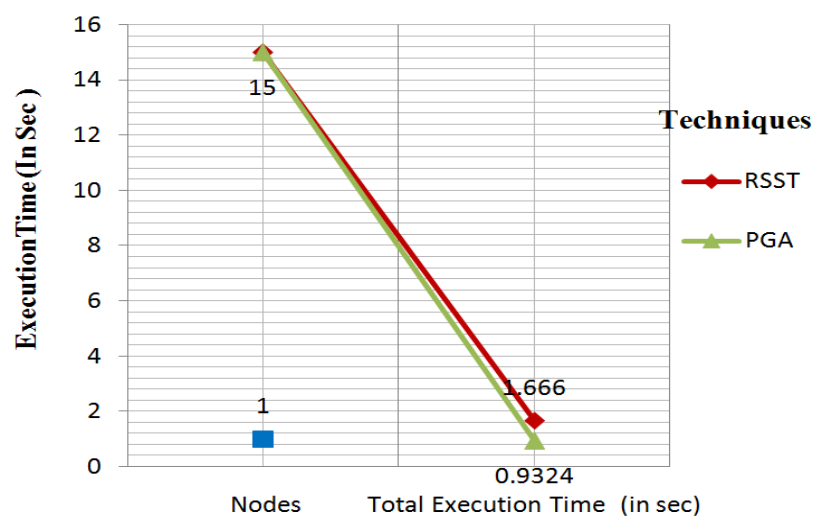

Fig3 Time Performance comparison between RSST and PGA in expertmention1: 15 nodes

In expertmention1 supported 15 nodes total execution time values compare RSST and PGA show result in graph and 


\author{
Vol. 8, Issue 6, June 2019
}

PGA realize total execution time 0.9324 and RSST realize total execution time one.666.overall realize PGA Technique best resolution in wireless device network.

\title{
VII. CONCLUSION
}

The projected secure localization schemes. It'll improve the positioning performance in WSN. Thus the distributed formula is very simple and it also can work. Though once the network is disconnected that is no international coordination is needed among nodes and this type of formula provides satisfactory accuracy. There are several directions for future ads WSN localization. Reducing localization latency is one in all the foremost necessary benchmarks for WSNs. Currently; an exchange exists between the speedy execution of associate formula and its accuracy. Additional work is needed that targeted on reducing run-time and error, whereas maintaining positioning accuracy. In addition, the majority of localization algorithms to the present purpose are centralized. For mobile detector localization, typically this can be this may be often a poor style various, as a result of the additional error and energy costs incurred. The event of further distributed localization techniques would be a welcome addition to WSN localization. There is a lot of interest in localization in urban and indoor areas where obstacles like vehicles, walls, people, and piece of furnishings cause multipath propagation and loss of line of sight. Most current ways in which use some variation of RSST identification, in conjunction with improvement techniques. However, new ways in which unit of measurement required as they expand mobile sensing to areas where training data cannot safely be obtained, like urban war zones or burning buildings. Lastly, mobile motivated sensors are presently being developed with motesized kind factors. Like embedded device nodes, these devices even have resource constraints, that limit their ability to navigate a sensing region among an equivalent suggests that an automaton with a full array of sensors and powerful method capability might. Proposed genetic algorithm (PGA) improved WSN in show result compare to RSST. They are able to expect to examine many advances in mobile device navigation among the on the point of future

\section{REFERENCES}

[1]. Z. Chaczko, R. Klempous, J. Nikodem and M. Nikodem, “Methods of sensors localization in wireless networks”, IEEE, 2007.

[2]. S. U. Sastry, N. \& D. Wagner, "Secure verification of location claims," in Proceedings of the $2^{\text {nd }}$ ACM workshop on Wireless sec, Sep 2003.

[3]. Y. Zeng, J. Cao, J. Hong, and L. Xie, "Secure localization and location verification in wireless sensor networks," in IEEE 6th International Conference on Mobile Adhoc and Sensor Systems, October 2009, pp. 864-869.

[4]. H. Wymeersch, J. Lien, and M. Z. Win, “Cooperative localization in wireless networks,” Proc. IEEE, vol. 97, no. 2, pp. 427-450, Feb. 2009.

[5]. A. H. Sayed, A. Tarighat, and N. Khajehnouri, "Network-based wireless location: Challenges faced in developing techniques for accurate wireless location information," IEEE Signal Process, Mag.,vol. 22, no. 4, pp. 24-40, Jul. 2005.

[6]. Niu, Ruixin, Aditya Vempaty, and Pramod K. Varshney. "Received-signal-strength-based localization in wireless sensor networks." Proceedings of the IEEE 106.7 (2018): 1166-1182.

[7]. Harikrishnan.G, "Minimizing Localization Error in Wireless Sensor Networks", International Journal of Advanced Information Science and Technology (IJAIST) ISSN: 2319:2682 Vol.2, No.10, October 2013.

[8]. Balasubramanian, S, \& R. Kavitha. "Minimizing the localization error in wireless sensor networks." Procedia engg 38 (2012): $3097-3104$.

[9]. Du, Wenliang, Lei Fang, and P. Ningi. "LAD: Localization anomaly detection for wireless sensor networks." 19th IEEE International Parallel and Distributed Processing Symposium. IEEE, 2005.

[10]. Sheu J.P, Chen P.C, and Hs u C.S, “A Dis tributed Localization Sche me for Wireles s Sensor Networks with Improved Grid-Scan and VectorBas ed Refine ment," IEEE Trans . Mobile Co mputing, 2008, Vol. 7, No. 9, pp. 1110 - 1123.

[11]. Md. Tareq Adnan, Suprakash Datta, Stuart MacLean, "Efficient and Accurate Range-based Sensor Network Localization", Procedia Computer Science ,405-413, 10,2012

[12]. Xinwei Wanga, Ole Bischoffa, Rainer Laura, Steffen Paula, "Localization in Wireless Ad-hoc Sensor Networks using Multilateration with RSSI for Logistic Applications”, Elsevier ,Available online 4 September , 461-464,2009.

[13]. B. riniva \& P.sangeetha, “Mobile Anchor-Based localization using Pso \& Path Planning Alg in Wireless Sensor Networks", IJIRAS, Vol.2, pp 5-8, 2015

[14]. Sung-Hwa Hong, Byoung-Kug Kim, and Doo-Seop Eom,"Localization Algorithm in Wireless Sensor Networks with Network Mobility" Manuscript received September 20, , IEEE,2009.

[15]. A. Kulaib, R. Shubair, M. Al-Qutayri, and J. W. Ng, "An overview of localization techniques for wireless sensor networks," in Innovations in Information Technology (IIT), International Conference on, pp. 167-172, IEEE, 2011.

[16]. Savvides, Andreas, Chih-Chieh Han, and Mani B. Strivastava. "Dynamic fine-grained localization in ad-hoc networks of sensors." In Proceedings of the 7th annual international conference on Mobile computing and networking, pp. 166-179. ACM, 2001.

[17]. Sharma, Meenakshi, and Anil Kumar Shaw. "Transmission time and throughput analysis of EEE LEACH, LEACH and direct transmission protocol: a simulation based approach." Advanced Computing 3, no. 6 (2012): 75.

[18]. Chaurasiya, Vijay K., Neeraj Jain, and Gora Chand Nandi. "A novel distance estimation approach for 3D localization in wireless sensor network using multi dimensional scaling." Information Fusion 15 (2014): 5-18.

[19]. Adewumi, Omotayo G., Karim Djouani, and Anish M. Kurien. "RSSI based indoor and outdoor distance estimation for localization in WSN." In 2013 IEEE international conference on Industrial technology (ICIT), pp. 1534-1539. IEEE, 2013.

[20]. Alippi, Cesare, \& Giovanni Vanini. "A RSSI-based and calibrated centralized localization technique for Wireless Sensor Networks." In Fourth Annual IEEE International Conference on Pervasive Computing and Communications Workshops (PERCOMW'06), pp. 5-pp. IEEE, 2006 